\title{
Avaliação Clínica da Aceitabilidade Cutânea de Creme Hidratante, Loção Hidratante e Sabonete Líquido Corporal em Peles Oncológicas
}

\author{
Clinical Evaluation of Cutaneous Acceptability of a moisturizing cream, a moisturizing lotion and a \\ liquid body soap in Oncological Skin
}

Dolores Gonzalez Fabra ${ }^{1}$, Karine Corcione Turke ${ }^{2}$, Jean Henri Maselli Schoueri², Vanessa Salzano ${ }^{1}$, Carlos D’Apparecida Machado Filho

Com citar: Fabra DG, Turke KC, Schoueri JHM, Salzano V, Machado CA Fo. Avaliação Clínica da

Aceitabilidade Cutânea de Creme Hidratante, Loção Hidratante e Sabonete Líquido Corporal em

Peles Oncológicas. Clin Onc Let. 2018;3(1-2):17-27. https://doi.org/10.4322/col.2018.001

\begin{abstract}
Resumo
Objetivo: Verificar a aceitabilidade cutânea em peles oncológicas dos produtos: Klaviê Creme, Klaviê Loção e Klaviê Sabonete Líquido. Métodos: Estudo clínico, com inclusão de 40 participantes em tratamento oncológico para uso contínuo das substâncias a serem testadas, por não menos que 21 dias. A seleção foi realizada através de prontuários clínicos e banco de dados formado pelo Centro de Estudos Dermatológicos Kenji Toyoda, em parceria com a médica investigadora principal. Os participantes foram submetidos à avaliação dermatológica clínica e testou-se a hidratação cutânea e perda de água transdérmica por equipamento de técnica não invasiva (CORNEOMETER ${ }^{\circledast}$ e TEWAMETER ${ }^{\circledast}$ da fabricante Courage-Khazaka) para medição das características fisiológicas da pele. Resultados: As aferições do Corneometer ${ }^{\circledR}$ demonstram eficácia hidratante em $85 \%$ dos participantes, com aumento de $38 \%$ no nível de hidratação da pele. Além disso, dos participantes avaliados por meio do Tewameter ${ }^{\circ}, 65 \%$ reduziram a perda de água da pele em média $14 \%$. Foram contabilizadas 06 perdas de seguimento e, apenas, 02 exclusões por evento adverso não grave não ligados ao produto, mas ao tratamento oncológico previamente estabelecido. Conclusão: Houve boa aceitabilidade cutânea dos produtos testados, tendo sido considerado aprovado para uso tópico desde que estritamente observadas as orientações de sua rotulagem no decorrer da aplicação, como orientado pelo fabricante.
\end{abstract}

Palavras-chave: Estudo clínico; Dermatologia; Oncologia

\begin{abstract}
Objective: To verify the cutaneous acceptability in oncological skin of the products: Klaviê Cream, Klaviê Lotion and Klaviê Liquid Soap. Methods: Clinical study, including 40 participants in cancer treatment for continuous use of the substances to be tested, for no less than 21 days. The selection was made through clinical records and database formed by the Kenji Toyoda Dermatological Studies Center, in partnership with the principal investigator. The participants were submitted to clinical dermatological evaluation and skin hydration was tested by non-evasive technique (CORNEOMETER ${ }^{\circledR}$ and TEWAMETER $^{\circledR}$ from manufacturer Courage -Khazaka) for the measurement of the physiological characteristics of the skin. Results: Corneometer $^{\circledR}$ measurements demonstrate hydrating efficacy in $85 \%$ of the participants, with a $38 \%$ increase in the level of skin hydration. In addition, of the participants evaluated using Tewameter ${ }^{\circledR}, 65 \%$ reduced skin water loss by an average of $14 \%$. There were 6 follow-up losses and only 2 exclusions per non-severe adverse event. Conclusion: There was good cutaneous acceptability of the tested products, having been considered approved for topical use provided strictly following of the guidelines of its labeling during the application, as directed by the manufacturer
\end{abstract}

Keywords: Clinical study; Dermatology; Oncology

\footnotetext{
${ }^{1}$ Departamento de Dermatologia. Faculdade de Medicina do ABC, Santo André, SP, Brasil

${ }^{2}$ Acadêmico. Faculdade de Medicina do ABC, Santo André, SP, Brasil

${ }^{3}$ Professor titular da Disciplina de Dermatologia. Faculdade de Medicina do ABC, Santo André, SP, Brasil

Financiamento: TheraSkin Farmaceutica Itda.

Conflito de interesse: Os autores declaram que o estudo foi patrocinado pela TheraSkin Farmaceutica Itda.
} 


\section{INTRODUÇÃO}

Nos últimos anos, com o avanço dos tratamentos oncológicos, percebemos um aumento importante da sobrevida dos pacientes com câncer além de uma superioridade em termos de segurança e eficácia dessas novas modalidades de tratamento que revolucionam o tratamento de tumores malignos hematológicos ou sólidos. Porém, apesar de demonstrarem uma maior tolerabilidade sistêmica, eles estão muito relacionados a efeitos adversos cutâneos o que acaba por impactar na sequência do tratamento oncológico e também acarretar morbidades que interferem de forma negativa na qualidade de vida desses pacientes. ${ }^{1}$

O prurido e a xerose cutânea são alguns exemplos da toxicidade desse tipo de tratamento sobre o tegumento dos pacientes. ${ }^{2}$

A xerose, além de ser uma preocupação psico-social, também é precursora de inúmeras complicações como prurido, predisposição a infecções, sensibilizações a alérgenos, todos decorrentes de uma quebra da barreira de proteção cutânea. $^{3}$

A radioterapia, outra modalidade terapêutica do arsenal de terapias oncológicos, desencadeia na pele um quadro denominado radiodermite que é uma reação inflamatória cutânea decorrente da exposição a radiação ionizante. Os sintomas podem aparecer durante ou mesmo após o término do tratamento podendo variar de um leve eritema (radiodermite grau I) até exulcerações importantes (radiodermite grau IV) que levam a interrupção do tratamento e incapacidades e limitações nos pacientes afetados. ${ }^{4}$

O diagnóstico e tratamento adequado dessas complicações são extremamente importantes para que não haja uma diminuição das doses dos medicamentos e até mesmo interrupção do tratamento oncológico proposto que podem impactar de forma negativa nos resultados do tratamento e taxas de sobrevida dos pacientes. ${ }^{5}$

Tendo em vista que o intuito principal dos produtos para cuidados da pele é promover um suporte exógeno para manter a barreira cutânea intacta, assim como preservar a hidratação da pele visando reduzir e aliviar os sintomas de xerose e consequentemente, prurido, infeções secundárias e reações de sensibilização, além de tornar a pele mais resistente aos efeitos da radiação, o objetivo do presente trabalho foi verificar a aceitabilidade cutânea em peles oncológicas dos produtos: Klaviê Creme, Klaviê Loção e Klaviê Sabonete Líquido por meio de observação da melhora na hidratação e redução da perda transepidérmica de água da pele em pacientes em tratamento oncológico (quimioterapia ou radioterapia). ${ }^{6}$

\section{MÉTODOS}

\section{DESENHO DE ESTUDO E PARTICIPANTES}

Trata-se de um estudo clínico, no qual foi determinado a inclusão de 40 (quarenta) participantes em tratamento oncológico (quimioterapia ou radioterapia) para uso contínuo das substâncias a serem testadas, por não menos que 21 dias. A seleção foi realizada através de prontuários clínicos e banco de dados formado pelo Centro de Estudos Dermatológicos Kenji Toyoda, em parceria com a médica investigadora principal DGF.

\section{CRITÉRIOS ESPECÍFICOS PARA INCLUSÃO, EXCLUSÃO E RESTRIÇÕES DO ESTUDO}

Os critérios específicos de inclusão, definidos pelo protocolo, foram: pacientes de ambos os sexos; fototipo (Fitzpatrick) de I a Vl; idade acima de 18 anos; tratamento oncológico por quimioterapia ou radioterapia.

Por sua vez, foram excluídos durante a fase de seleção os participantes que apresentaram quaisquer das seguintes características: queimadura grave exsudativa; alergia a algum dos componentes da fórmula; alteração de cognição; pós-cirúrgico de menos de 30 dias; infecção no local (determinado pelo pesquisador); estar em uso de, ou ter utilizado qualquer creme ou medicamento tópico no local em até 7 dias antes do início do estudo; presença de qualquer condição que, na opinião da médica investigadora, possa interferir no resultado ou seja um risco ao projeto e/ou ao participante.

Por fim, o presente estudo apresentou como restrições: necessidade de wash out de 7 dias para hidratantes ou medicamentos tópicos e/ou por via oral com potencial para intervir na avaliação da pele; não usar chás, compressas, shampoos e sabonetes específicos para pele oncológica durante o banho; nenhuma aplicação de outro produto da mesma categoria (além daquele em teste) na área experimental; ao utilizar medicamento fora dos padrões de tratamento oncológico, informar a pesquisadora responsável. Além disso, o participante não pôde se expor ao sol 
ou lâmpadas UV durante o estudo, nem estar em tratamento com antialérgicos, anti-inflamatórios (sistêmico ou corticoides tópicos) durante o período do teste. Como restrições finais, elencam-se: qualquer tratamento que interfira no resultado do estudo; estar participando de outro estudo clínico.

\section{ÁREA E CONDIÇÕES DE APLICAÇÃO DO PRODUTO EM ESTUDO}

O ensaio clínico envolveu o uso em conjunto de 03 (três) produtos da linha Klaviê: loção, creme e sabonete líquido. De acordo com o modo de uso recomendados pelo fabricante/patrocinador:

- Sabonete: usar durante o banho ou limpeza diária do rosto e corpo. Aplicar sobre a pele úmida e massagear suavemente antes de enxaguar.

- Loção: aplicar no corpo, preferencialmente após o banho. Repetir a aplicação sempre que necessário ou a critério médico.

- Creme: aplicar na pele seca e áreas mais ressecadas, preferencialmente após o banho. Repetir a aplicação sempre que necessário ou a critério médico.

\section{AVALIAÇÃO DERMATOLÓGICA}

As reações do órgão cutâneo-mucoso às noxas traduzem-se por número limitado de respostas morfológicas e que constituem lesões elementares. As lesões elementares são classificadas em cinco grupos bem definidos. A interpretação dos resultados do exame cutâneo e do caderno do investigador é aquela definida para este tipo de estudo conforme o procedimento correspondente classificado de acordo com a intensidade das reações, conforme a Tabela 1.

Tabela 1. Interpretação do exame cutâneo

\begin{tabular}{|c|c|}
\hline ALTERAÇÕES DE COR & $\begin{array}{ll}\text { MÁCULAS VÁSCULO SANGUINEAS } & \text { MÁCULAS PIGMENTARES OU DISCROMIAS } \\
\text { Eritema: por vasodilatação } & \text { Hipercromia: cor variável por aumento de melanina ou } \\
\text { Púrpura: mancha vermelha que não desaparece à } & \text { outros pigmentos } \\
\text { vitropressão. } & \text { Hipocromia: alteração de cor em decorrência da } \\
& \text { diminuição da melanina. }\end{array}$ \\
\hline FORMAÇÕES SÓLIDAS & $\begin{array}{l}\text { RESULTAM DE PROCESSOS INFLAMATÓRIOS ATINGINDO, ISOLADA OU CONJUNTAMENTE A EPIDERME, } \\
\text { DERME E A HIPODERME } \\
\text { Pápula: lesão sólida, circunscrita, elevada, menor que } 1 \mathrm{~cm} \text { de tamanho, por processo patológico epidérmico, } \\
\text { dérmico ou misto. }\end{array}$ \\
\hline COLEÇÕES LÍQUIDAS & $\begin{array}{l}\text { NO GRUPO DAS COLEÇÕES LÍQUIDAS, INCLUEM-SE AS LESÕES COM CONTEÚDO LÍQUIDO QUE PODE SER } \\
\text { SEROSIDADE, SANGUE OU PUS } \\
\text { Vesícula: elevação circunscrita até } 1 \mathrm{~cm} \text { de tamanho, contendo líquido claro; } \\
\text { Bolha: elevação circunscrita contendo líquido claro, maior que } 1 \mathrm{~cm} ; \\
\text { Pústula: elevação circunscrita até } 1 \mathrm{~cm} \text {, contendo pus. }\end{array}$ \\
\hline $\begin{array}{l}\text { ALTERAÇÕES DE } \\
\text { ESPESSURA }\end{array}$ & $\begin{array}{l}\text { Edema: aumento de espessura, depressível, com a cor própria da pele, por extravasamento de plasma na } \\
\text { derme e/ou hipoderme; } \\
\text { Liquenificação: espessamento da pele com acentuação dos sulcos e da cor própria, com aspecto } \\
\text { quadriculado. }\end{array}$ \\
\hline PERDAS E REPARAÇÕES & $\begin{array}{l}\text { LESÕES ORIUNDAS DA ELIMINAÇÃO OU DESTRUIÇÃO PATOLÓGICAS E DE REPARAÇÕES DE TECIDOS } \\
\text { CUTÂNEOS } \\
\text { Escama: massa furfurácea, massa furfurácea, micácea ou foliácea, que se desprende da superfície cutânea, } \\
\text { por alteração de queratinização; } \\
\text { Erosão: perda superficial, somente da epiderme; } \\
\text { Escoriação: erosão traumática, geralmente por coçagem; } \\
\text { Ulceração: perda circunscrita de epiderme e derme, podendo atingir a hipoderme e tecidos subjacentes; } \\
\text { Crosta: concreção de cor amarelo-clara, esverdeada ou vermelha escura, que se forma em área de } \\
\text { perda tecidual. }\end{array}$ \\
\hline
\end{tabular}




\section{AVALIAÇÃO INSTRUMENTAL}

Medida simultânea exata de características fisiológicas da pele com valores físicos para registro da média e passível de comparação inicial e final através de equipamento de método não invasivo. Os parâmetros hidratação e perda de água da pele são obtidos a partir de leitura por sonda que quantifica em números o nível de cada atributo.

Os participantes submetidos a avaliação da hidratação da pele por equipamento de técnica não invasiva (CORNEOMETER ${ }^{\circledast}$ e TEWAMETER ${ }^{\circledR}$ da fabricante Courage-Khazaka) para medição das características fisiológicas da pele, permaneceram em ambiente com temperatura e umidade controlados a $20^{\circ} \mathrm{C}$ e $40-60 \%$ de umidade e realizaram limpeza e assepsia da pele com água e sabonete neutro.

A determinação da hidratação foi interpretada de acordo com os resultados do instrumento Corneometer ${ }^{\circledR} .0$ mesmo mediu a profundidade de hidratação da pele em $30 \mu \mathrm{m}$; a camada córnea em sua espessura apresenta-se entre 15-20 $\mu \mathrm{m}$, portanto determinando que, para ser classificado como produto de hidratação o equipamento deve acusar aumento no nível da hidratação da pele (epiderme). Por sua vez, a determinação para a perda de água transepidérmica foi interpretada de acordo com os resultados do instrumento TEWAMETER ${ }^{\circledR}$. A avaliação instrumental encontra-se detalhada nas Tabelas 2 e 3.

Tabela 2. Avaliação Instrumental referente à Hidratação

\begin{tabular}{|c|c|c|c|c|}
\hline \multicolumn{5}{|c|}{ Hidratação } \\
\hline Ref. & LOCAL DE APLICAÇÃO/AVALIAÇÃO INSTRUMENTAL & DO & D30 & \% DE DIFERENÇA \\
\hline 1 & PESCOÇO & 0,0 & 0,0 & 0,0 \\
\hline 2 & PESCOÇO & 62,4 & 87,6 & 25,2 \\
\hline 3 & FACE & 0,0 & 0,0 & 0,0 \\
\hline 4 & MAMA & 0,0 & 0,0 & 0,0 \\
\hline 5 & PÉS & 38,5 & 67,4 & 28,9 \\
\hline 6 & FACE & 62,0 & 62,5 & 0,5 \\
\hline 7 & FACE & 37,1 & 50,5 & 13,4 \\
\hline 8 & PERNAS & 16,6 & 69,5 & 52,9 \\
\hline 9 & MAMA & 0,0 & 0,0 & 0,0 \\
\hline 10 & FACE & 67,0 & 81,0 & 14,0 \\
\hline 11 & BRAÇOS & 20,8 & 59,8 & 39,0 \\
\hline 12 & BRAÇOS & 0,0 & 0,0 & 0,0 \\
\hline 13 & BRAÇOS & 50,9 & 61,8 & 10,9 \\
\hline 14 & BRAÇOS & 46,0 & 46,0 & 0,0 \\
\hline 15 & PERNAS & 40,8 & 76,0 & 35,2 \\
\hline 16 & ROSTO & 26,2 & 75,6 & 49,4 \\
\hline 17 & DORSO & 31,0 & 66,2 & 35,2 \\
\hline 18 & BRAÇOS & 45,3 & 65,0 & 19,7 \\
\hline 19 & PERNAS & 49,5 & 66,6 & 17,1 \\
\hline 20 & FACE & 39,9 & 53,3 & 13,4 \\
\hline 21 & COLO & 32,1 & 69,4 & 37,3 \\
\hline 22 & MAMAS & 55,4 & 55,5 & 0,1 \\
\hline 23 & BRAÇOS & 37,6 & 49,1 & 11,5 \\
\hline 24 & BRAÇOS & 77,3 & 71,5 & $-5,8$ \\
\hline 25 & FACE & 50,3 & 61,7 & 11,4 \\
\hline 26 & MAMA & 37,8 & 65,4 & 27,6 \\
\hline 27 & PERNAS & 41,5 & 47,8 & 6,3 \\
\hline 28 & BRAÇO & 0,0 & 0,0 & 0,0 \\
\hline 29 & MAMA & 55,0 & 53,7 & $-1,3$ \\
\hline
\end{tabular}


Tabela 2. Continuação...

\begin{tabular}{ccccc}
\hline & & Hidratação & & \\
\hline Ref. & LOCAL DE APLICAÇÃO/AVALIAÇÃO INSTRUMENTAL & D0 & D30 & \% DE DIFERENÇA \\
\hline 30 & MAMA & 46,2 & 48,9 & 2,7 \\
31 & MAMA & 73,1 & 73,1 & 0,0 \\
32 & PERNAS & 40,6 & 48,3 & 7,7 \\
33 & BRAÇO & 50,7 & 50,7 & 0,0 \\
34 & BRAÇO & 55,7 & 79,6 & 23,9 \\
35 & FACE & 0,0 & 0,0 & 0,0 \\
36 & BRAÇO ESQUERDO & 42,9 & 60,9 & 18,0 \\
37 & FACE & 44,2 & 53,7 & 9,5 \\
39 & PERNAS & 49,2 & 80,4 & 31,2 \\
40 & PERNAS & 0,0 & 0,0 & 0,0 \\
& BRAÇO DIREITO & 40,8 & 62,0 & 21,2 \\
\end{tabular}

Tabela 3. Avaliação Instrumental referente ao TEWL ${ }^{\circledR}$

\begin{tabular}{|c|c|c|c|c|}
\hline \multicolumn{5}{|c|}{ TEWL } \\
\hline Ref. & LOCAL DE APLICAÇÃO/AVALIAÇÃO INSTRUMENTAL & DO & D30 & \% DE DIFERENÇA \\
\hline 1 & PESCOÇO & 0,0 & 0,0 & 0,0 \\
\hline 2 & PESCOÇO & 9,9 & 9,0 & 0,9 \\
\hline 3 & FACE & 0,0 & 0,0 & 0,0 \\
\hline 4 & MAMA & 0,0 & 0,0 & 0,0 \\
\hline 5 & PÉS & 22,9 & 5,1 & 17,8 \\
\hline 6 & FACE & 7,2 & 8,5 & $-1,3$ \\
\hline 7 & FACE & 8,3 & 13,5 & $-5,2$ \\
\hline 8 & PERNAS & 9,6 & 4,6 & 5,0 \\
\hline 9 & MAMA & 0,0 & 0,0 & 0,0 \\
\hline 10 & FACE & 8,5 & 8,7 & $-0,2$ \\
\hline 11 & BRAÇOS & 11,0 & 9,7 & 1,3 \\
\hline 12 & BRAÇOS & 0,0 & 0,0 & 0,0 \\
\hline 13 & BRAÇOS & 11,3 & 10,1 & 1,2 \\
\hline 14 & BRAÇOS & 28,6 & 28,6 & 0,0 \\
\hline 15 & PERNAS & 10,2 & 9,9 & 0,3 \\
\hline 16 & ROSTO & 16,2 & 10,2 & 6,0 \\
\hline 17 & DORSO & 17,6 & 20,7 & $-3,1$ \\
\hline 18 & BRAÇOS & 18,0 & 10,2 & 7,8 \\
\hline 19 & PERNAS & 20,4 & 29,2 & $-8,8$ \\
\hline 20 & FACE & 16,8 & 12,8 & 4,0 \\
\hline 21 & COLO & 12,4 & 11,6 & 0,8 \\
\hline 22 & MAMAS & 15,2 & 10,1 & 5,1 \\
\hline 23 & BRAÇOS & 5,8 & 11,2 & $-5,4$ \\
\hline
\end{tabular}


Tabela 3. Continuação...

\begin{tabular}{|c|c|c|c|c|}
\hline \multicolumn{5}{|c|}{ TEWL } \\
\hline Ref. & LOCAL DE APLICAÇÃO/AVALIAÇÃO INSTRUMENTAL & DO & D30 & \% DE DIFERENÇA \\
\hline 24 & BRAÇOS & 10,6 & 4,9 & 5,7 \\
\hline 25 & FACE & 11,1 & 10,6 & 0,5 \\
\hline 26 & MAMA & 6,3 & 11,7 & $-5,4$ \\
\hline 27 & PERNAS & 10,5 & 13,0 & $-2,5$ \\
\hline 28 & BRAÇO & 0,0 & 0,0 & 0,0 \\
\hline 29 & MAMA & 5,3 & 12,0 & $-6,7$ \\
\hline 30 & MAMA & 8,8 & 10,4 & $-1,6$ \\
\hline 31 & MAMA & 3,1 & 3,1 & 0,0 \\
\hline 32 & PERNAS & 43,3 & 12,0 & 31,3 \\
\hline 33 & BRAÇO & 11,6 & 11,6 & 0,0 \\
\hline 34 & BRAÇO & 14,1 & 8,9 & 5,2 \\
\hline 35 & FACE & 0,0 & 0,0 & 0,0 \\
\hline 36 & BRAÇO ESQUERDO & 17,0 & 15,0 & 2,0 \\
\hline 37 & FACE & 13,0 & 11,0 & 2,0 \\
\hline 38 & PERNAS & 15,5 & 11,5 & 4,0 \\
\hline 39 & PERNAS & 0,0 & 0,0 & 0,0 \\
\hline \multirow[t]{2}{*}{40} & BRAÇO DIREITO & 10,1 & 9,3 & 0,8 \\
\hline & Média & 10,76 & 9,22 & -14 \\
\hline
\end{tabular}

\section{DINÂMICA DO ESTUDO}

O presente estudo foi compreendido por duas avaliações distintas, realizadas em dois momentos diferentes durante a primeira e a segunda visita, respectivamente. Uma breve descrição de cada etapa encontra-se abaixo:

Visita 01: Formalização da ficha com dados cadastrais (contatos e documentos dos participantes da pesquisa). Logo em seguida foi entregue o Termo de Consentimento Livre e Esclarecido (TCLE). O TCLE foi lido em voz alta e após compreendido e sanadas eventuais dúvidas foi assinado, datado e rubricado em todas as folhas com a retenção de uma via pelo Centro de Pesquisa e outra entregue ao participante. A Investigadora principal fez a abertura do prontuário clínico para confirmar os critérios de inclusão e exclusão definidos em protocolo. $O$ médico dermatologista iniciou os procedimentos de preenchimento da ficha clínica do participante e inclusão do mesmo no caderno do investigador. Após a inclusão, o participante recebeu instruções para preenchimento do diário de uso para relatos de eventos adversos ou quaisquer observações geradas durante o uso do produto. Cada participante foi orientado sobre tipos de irritações e eventos adversos.

Em uma sala de climatização para medidas de hidratação da pele, o participante foi submetido ao período de aclimatação obrigatório. A medida de hidratação da pele é realizada por sonda Corneometer $^{\circledR}$, da fabricante Courage-Khazaka, por meio de sistema capacitivo e método não invasivo e a medida da perda transepidérmica de água da pele é mensurada pelo Tewameter $^{\circledR}$ - Courage-Khazaka. O participante recebeu as instruções finais, sendo orientado a visita de retorno e aos contatos de emergências, caso necessário. Foi feito o ressarcimento dos valores referentes à locomoção até o Centro de Pesquisa.

Visita 02: A visita de retorno foi iniciada com a avaliação dermatológica pela médica investigadora, onde foi questionado sobre eventos adversos e anotações registradas em diário de uso. Todas as informações foram registradas no caderno do investigador. O participante foi encaminhado ao período de aclimatação para indicação das medidas instrumentais de hidratação da pele. O participante foi ressarcido com o pagamento da locomoção ao Centro de Pesquisa.

\section{ASPECTOS ÉTICOS}

A avaliação clínica de segurança foi precedida da análise da formulação, denominada "Avaliação Pré-clínica", que compreende: 
1. Avaliação do produto final ou ingrediente objeto de avaliação de segurança, quanto a parâmetros de caracterização, finalidade e aplicação compreendida na definição de cosméticos estabelecida pela resolução ANVISA RDC $n^{\circ} 07$ DE FEVEREIRO DE 2015;

2. Determinação prévia do potencial de risco do produto, considerando-se as informações técnicas prestadas pelo fabricante e/ou importador do produto ou matéria-prima;

3. Avaliação do potencial de reações adversas devido ao uso inadequado ou acidental do produto.

$O$ estudo foi fundamentado em documentos nacionais e internacionais que emanam declarações e diretrizes que envolvem pesquisas conduzidas em humanos. De acordo com Resolução 466/12 do Ministério da Saúde, Boas Práticas Clínicas (Documento das Américas e ICH E6: Good Clinical Practice).

Antes do início da pesquisa um Termo de Consentimento Livre e Esclarecido (TCLE), foi elaborado de acordo com as normas, e aprovado pelo Comitê de Ética em Pesquisa da Faculdade de Medicina do ABC, pelo Pareceres Consubstanciados sob os números: № 2.274 .312 e 2.584.152. Todos os participantes assinaram o Termo de Consentimento Livre e Esclarecido (TCLE) em duas vias.

\section{RESULTADOS}

Foram selecionados 40 participantes da pesquisa, desses 11 são homens e 29 são mulheres. Dos 32 (trinta e dois) participantes que concluíram o estudo, alguns realizaram apenas a terapia de quimioterapia, radioterapia ou ambas as terapias, descrita no Apêndice A. Os resultados indicados pelo equipamento Corneometer ${ }^{\circledR}$ demonstram eficácia hidratante em $85 \%$ dos participantes, com aumento médio de $38 \%$ no nível de hidratação da pele. Assim, o produto foi classificado como hidratante, pois apresentou nível de hidratação maior que a avaliação inicial.

Além disso, dos participantes avaliados por meio do Tewameter $^{\circledR}, 65 \%$ reduziram a perda de água da pele em média $14 \%$. Desse modo, o produto auxiliou na redução da perda de água da pele, refletindo uma melhora na barreira cutânea - os dados numéricos apresentam-se menores em comparação a avaliação inicial.

Percorridos os 21 dias de estudo, foram contabilizados 06 perdas de seguimento e 02 exclusões por evento adverso não grave. Com relação às perdas de seguimento, nenhum dos seis pacientes em questão retornou para a Visita 02. Foram realizados contatos telefônicos até obtenção de um respaldo dos mesmos, que, por indisponibilidade de horário, não puderam retornar à visita final. O evento adverso relacionado ao uso do produto foi notificado ao CEP e reportado ao patrocinador no prazo estabelecido. Os demais eventos não foram relacionados ao uso dos produtos.

\section{DISCUSSÃO}

Apesar do aumento do número de casos de pacientes diagnosticados com câncer nos últimos anos, a sobrevida destes pacientes vem aumentando significativamente devido aos novos tratamentos. Durante esse processo, no entanto, os pacientes experimentam um alto grau de sofrimento, agravado por alterações dermatológicas e estéticas. Em alguns casos, as terapias podem ser agressivas a ponto de o enfermo precisar abandonar o tratamento. Esse fato reforça a importância do suporte dermato-cosmiátrico ao longo de todo o processo, através de produtos que promovam adequada hidratação na pele do paciente para minimizar o impacto das terapêuticas contra o câncer, tanto na quimioterapia, imunoterapia, terapia-alvo e radioterapia. Outro estudo já demonstrou que o uso de agentes tópicos em pacientes que passaram por tratamento radioterápico pode ser mais indicado que o tratamento expectante. $^{7}$

No presente estudo, observamos através da avaliação clínica do produto estudado, diminuição dos processos inflamatórios locais na pele, decorrentes do tratamento de radioterapia, diminuição da ardência local relatado por grande parte dos pacientes, clareamento da hipercromia pós-inflamatória local, hidratação importante mesmo durante o tratamento oncológico e boa aceitação pelos pacientes do aspecto sensorial do produto.

Foi observado através das avaliações instrumentais, uma melhora significativa da hidratação da pele e redução da perda transepidérmica de água, mesmo durante as diferentes terapêuticas oncológicas às quais os pacientes estudados estavam sendo submetidos. 
O produto apresenta eficácia significativa para prevenção da xerose e melhora da barreira cutânea e garante a adequada hidratação da pele mesmo durante os tratamentos oncológicos.

\section{CONCLUSÃO}

Nas condições em que os produtos Klaviê Sabonete Líquido; Klaviê Loção Hidratante e Klaviê Creme Hidratante, da empresa THERASKIN FARMACÊUTICA LTDA foram testados, os mesmos apresentaram boa aceitabilidade cutânea, tendo sido considerados aprovados para uso tópico para pacientes em tratamento oncológico desde que estritamente observadas as orientações de sua rotulagem no decorrer da aplicação, como orientado pelo fabricante.

Este estudo, de acordo com o Guia para Avaliação de Segurança de Produtos Cosméticos da Agência de Vigilância Sanitária / Ministério da Saúde, dá sustentação aos dizeres na embalagem do produto: "Dermatologicamente testado em peles oncológicas"; "Produto para pele oncológica"; "Produto hidratante para pele oncológica"; "Produto umectante"; "Mantém hidratação da pele"; "Manutenção do manto lipídico"; "previne a perda de água da pele". Estudos de eficácia dos produtos já estão em andamento.

\section{REFERÊNCIAS}

1. Burtness B, Anadkat M, Basti S, et al. NCCN task force report: management of dermatologic and other toxicities associated with EGFR inhibition in patients with cancer. J Natl Compr Canc Netw. 2009;7(Suppl 1):5-24. http://dx.doi.org/10.6004/jnccn.2009.0074. PMid:19470276.

2. Fischer A, Rosen AC, Ensslin CJ, Wu S, Lacouture ME. Pruritus to anticancer agents targeting the EGFR, BRAF, and CTLA-4. Dermatol Ther. 2013;26(2):135-48. http://dx.doi.org/10.1111/dth.12027. PMid:23551370.

3. Valentine J, Belum VR, Duran J, et al. Incidence and risk of xerosis with targeted anticancer therapies. J Am Acad Dermatol. 2015;656(4):72. PMid:25637330.

4. Sekiguchi K, Akahane K, Ogita M, et al. Efficacy of heparinoid moisturizer as a prophylactic agent for radiation dermatitis following radiotherapy after breast-conserving surgery: a randomized controlled trial. Jpn J Clin Oncol. 2018;48(5):450-7. http://dx.doi.org/10.1093/jjco/hyy045. PMid:29635534.

5. Skolarus TA, Holmes-Rovner M, Northouse LL, et al. Primary care perspectives on prostate cancer survivorship: implications for improving quality of care. Urol Oncol. 2013;31(6):727-32.

http://dx.doi.org/10.1016/j.urolonc.2011.06.002. PMid:21775171.

6. Dreno B, Bensadoun RJ, Humbert $P$, et al. Algorithm for dermocosmetic use in the management of cutaneous sideeffects associated with targeted therapy in oncology. J Eur Acad Dermatol Venereol. 2013;27(9):1071-80.

http://dx.doi.org/10.1111/jdv.12082. PMid:23368717.

7. Baumann BC, Verginadis II, Zeng C, et al. Assessing the validity of clinician advice that patients avoid use of topical agents before daily radiotherapy treatments. JAMA Oncol. 2018;4(12):1742-8.

http://dx.doi.org/10.1001/jamaoncol.2018.4292. PMid:30347008. 
Apêndice A. Características gerais dos participantes.

\begin{tabular}{|c|c|c|c|c|c|c|c|c|}
\hline Prontuário & INICIAIS & SEXO & IDADE & FOTOTIPO & NEOPLASIA & $\begin{array}{c}\text { TRATAMENTO } \\
\text { ANTI-NEOPLASICO }\end{array}$ & AFECÇÕES CUTÂNEAS & $\begin{array}{l}\text { PERCURSO } \\
\text { DO } \\
\text { ESTUDO }\end{array}$ \\
\hline 1 & J.LEIT & M & 69 & III & Câncer de Laringe & $Q t+R t$ & $\begin{array}{l}\text { Erupção e eritema em } \\
\text { região cervical e } \\
\text { torácica anterior; } \\
\text { pústulas no dorso; } \\
\text { radiodermite; erupção } \\
\text { acneiforme; xerose }\end{array}$ & EX \\
\hline 2 & D.SANT & $\mathrm{F}$ & 74 & IV & Câncer de Laringe & $\mathrm{Qt}+\mathrm{Rt}$ & $\begin{array}{l}\text { Radiodermite em } \\
\text { região cervical anterior }\end{array}$ & $\begin{array}{l}37 \text { dias de } \\
\text { uso }\end{array}$ \\
\hline 3 & J.SANT & M & 61 & IV & Câncer de Cólon & Qt & $\begin{array}{c}\text { Xerose cutânea; } \\
\text { hipercromia linear no } \\
\text { trajeto da } \\
\text { quimioterapia }\end{array}$ & PS \\
\hline 4 & M.ALM & $\mathrm{F}$ & 43 & IV & Câncer de Mama & $\mathrm{Qt}+\mathrm{Rt}$ & $\begin{array}{l}\text { Ptiríase versicolor; } \\
\text { eflúvio anágeno; } \\
\text { rarefação de supercílio; } \\
\text { hipercromia em virilha }\end{array}$ & PS \\
\hline 5 & C.COST & $\mathrm{F}$ & 42 & III & Câncer de Mama & $\mathrm{Qt}+\mathrm{Rt}+$ Cirurgia & $\begin{array}{l}\text { Tinea Pedis; Sd. Mão- } \\
\text { Pé; xerose cutânea; } \\
\text { hiperqueratose do } \\
\text { cotovelo; radiodermite } \\
\text { grau II; onicomicose }\end{array}$ & $\begin{array}{l}37 \text { dias de } \\
\text { uso }\end{array}$ \\
\hline 6 & S.SOUZ & $\mathrm{F}$ & 66 & II & Câncer de Cólon & $\mathrm{Qt}+\mathrm{Ht}$ & $\begin{array}{l}\text { Eritema; cisto folicular; } \\
\text { hipercromia de face e } \\
\text { membros superiores; } \\
\text { dermatite de contato }\end{array}$ & $\begin{array}{l}37 \text { dias de } \\
\text { uso }\end{array}$ \\
\hline 7 & C.SANT & $\mathrm{F}$ & 66 & III & Câncer de Mama & $Q t+R t$ & $\begin{array}{l}\text { Radiodermite; } \\
\text { melasma; eflúvio } \\
\text { anágeno; onicolise; } \\
\text { xerose cutânea }\end{array}$ & $\begin{array}{l}37 \text { dias de } \\
\text { uso }\end{array}$ \\
\hline 8 & L.BRAN & $\mathrm{F}$ & 59 & II & $\begin{array}{c}\text { Câncer de Intestino } \\
\text { Delgado }\end{array}$ & Qt + Cirurgia & $\begin{array}{l}\text { Xerose cutânea; } \\
\text { eczema numular em } \\
\text { dorso; mastite }\end{array}$ & $\begin{array}{l}37 \text { dias de } \\
\text { uso }\end{array}$ \\
\hline 9 & A.TELE & $\mathrm{F}$ & 45 & I & Câncer de Mama & $\mathrm{Qt}+\mathrm{Rt}+\mathrm{Ht}$ & $\begin{array}{c}\text { Exantema; lesões } \\
\text { aceniformes; dermatite } \\
\text { de contato }\end{array}$ & EX \\
\hline 10 & F.CARV & $\mathrm{F}$ & 48 & II & Câncer de Mama & $\mathrm{Qt}+\mathrm{Rt}+$ Cirurgia $+\mathrm{Ht}$ & $\begin{array}{c}\text { Melasma; erupção } \\
\text { acneiforme; cicatriz } \\
\text { hipertrófica; prurido }\end{array}$ & $\begin{array}{l}37 \text { dias de } \\
\text { uso }\end{array}$ \\
\hline 11 & V.ROSS & M & 62 & IV & Câncer de Esôfago & $\mathrm{Qt}+\mathrm{Rt}$ & $\begin{array}{l}\text { Queratose seborreica; } \\
\text { melanose; xerose } \\
\text { cutânea; onicomicose; } \\
\text { eczema; tinea pedis; } \\
\text { cisto folicular no dorso }\end{array}$ & $\begin{array}{l}37 \text { dias de } \\
\text { uso }\end{array}$ \\
\hline 12 & A.SOUZ & $\mathrm{F}$ & 64 & II & Câncer de Mama & Cirurgia + Qt & Xerose cutânea & PS \\
\hline 13 & M.MATT & $\mathrm{F}$ & 51 & II & Câncer de Mama & Qt & $\begin{array}{l}\text { Prurigo; hipercromia } \\
\text { residual }\end{array}$ & $\begin{array}{l}21 \text { dias de } \\
\text { uso }\end{array}$ \\
\hline 14 & L.BAPT & $\mathrm{F}$ & 62 & II & $\begin{array}{c}\text { Câncer de Mama + } \\
\text { Metástase óssea em } \\
\text { coluna }\end{array}$ & $\mathrm{Qt}+\mathrm{Rt}$ & Xerose cutânea & $\begin{array}{c}35 \text { dias de } \\
\text { uso }\end{array}$ \\
\hline
\end{tabular}




\begin{tabular}{|c|c|c|c|c|c|c|c|c|}
\hline Prontuário & INICIAIS & SEXO & IDADE & FOTOTIPO & NEOPLASIA & $\begin{array}{c}\text { TRATAMENTO } \\
\text { ANTI-NEOPLASICO }\end{array}$ & AFECÇÕES CUTÂNEAS & $\begin{array}{l}\text { PERCURSO } \\
\text { DO } \\
\text { ESTUDO }\end{array}$ \\
\hline 15 & A.DIAS & $M$ & 65 & IV & Câncer de Esôfago & $Q t+R t$ & $\begin{array}{l}\text { Xerose; radiodermite; } \\
\text { impetigo; onicomicose; } \\
\text { prurido }\end{array}$ & $\begin{array}{c}35 \text { dias de } \\
\text { uso }\end{array}$ \\
\hline 16 & S.DIAS & $\mathrm{F}$ & 58 & III & Câncer de Intestino & Cirurgia + Qt + Rt & $\begin{array}{c}\text { Melanose; } \\
\text { esclerodemia; xerose } \\
\text { cutânea }\end{array}$ & $\begin{array}{l}35 \text { dias de } \\
\text { uso }\end{array}$ \\
\hline 17 & S.CRUZ & M & 76 & IV & Mieloma Múltiplo & Qt & $\begin{array}{c}\text { Xerose cutânea; } \\
\text { vitiligo; eritema em } \\
\text { face }\end{array}$ & $\begin{array}{l}35 \text { dias de } \\
\text { uso }\end{array}$ \\
\hline 18 & S.ALVE & $\mathrm{F}$ & 46 & II & $\begin{array}{l}\text { Câncer de Mama + } \\
\text { Metástase óssea em } \\
\text { coluna }\end{array}$ & $Q t+R t$ & Xerose cutânea & $\begin{array}{l}35 \text { dias de } \\
\text { uso }\end{array}$ \\
\hline 19 & E.SEGU & $M$ & 61 & IV & $\begin{array}{c}\text { Linfoma Cutâneo de } \\
\text { Células T }\end{array}$ & Qt & $\begin{array}{c}\text { Prurido; placa } \\
\text { hipercrômica; } \\
\text { onicomicose; tinea- } \\
\text { pedis }\end{array}$ & $\begin{array}{l}35 \text { dias de } \\
\text { uso }\end{array}$ \\
\hline 20 & M.OLIV & $\mathrm{F}$ & 46 & II & Câncer de Mama & $Q t+R t$ & $\begin{array}{l}\text { Prurido; xerose } \\
\text { cutânea }\end{array}$ & $\begin{array}{l}35 \text { dias de } \\
\text { uso }\end{array}$ \\
\hline 21 & V.ASSI & $\mathrm{F}$ & 37 & II & Câncer de Mama & $\mathrm{Qt}+\mathrm{Rt}+\mathrm{Ht}$ & $\begin{array}{c}\text { Xerose cutânea; } \\
\text { hipercromia na área da } \\
\text { radioterapia; } \\
\text { hipercromia em virilha; } \\
\text { melasma }\end{array}$ & $\begin{array}{c}35 \text { dias de } \\
\text { uso }\end{array}$ \\
\hline 22 & M.SOUZ & $\mathrm{F}$ & 61 & II & Câncer de Mama & $\mathrm{Qt}+\mathrm{Rt}+$ Cirurgia $+\mathrm{Ht}$ & $\begin{array}{l}\text { Ressecamento } \\
\text { cutâneo; eflúveo } \\
\text { telógeno }\end{array}$ & $\begin{array}{l}35 \text { dias de } \\
\text { uso }\end{array}$ \\
\hline 23 & E.BATI & $\mathrm{F}$ & 63 & III & Câncer de Mama & $\mathrm{Qt}+\mathrm{Rt}+\mathrm{Ht}$ & Farmacodermia & $\begin{array}{l}28 \text { dias de } \\
\text { uso }\end{array}$ \\
\hline 24 & A.NAKA & $M$ & 67 & III & Timoma & $Q t+R t$ & $\begin{array}{c}\text { Dermatite; xerose } \\
\text { cutânea }\end{array}$ & $\begin{array}{l}28 \text { dias de } \\
\text { uso }\end{array}$ \\
\hline 25 & E.ELID & M & 54 & V & Câncer de Cólon & Qt & $\begin{array}{l}\text { Furúnculo; herpes } \\
\text { simples labial; } \\
\text { oninfecção; } \\
\text { onicomicose; erisipela }\end{array}$ & $\begin{array}{l}28 \text { dias de } \\
\text { uso }\end{array}$ \\
\hline 26 & M.CAMP & $\mathrm{F}$ & 65 & II & Câncer de Mama & Rt + Cirurgia & Xerose cutânea & $\begin{array}{c}21 \text { dias de } \\
\text { uso }\end{array}$ \\
\hline 27 & A.OLIV & $\mathrm{F}$ & 59 & III & Câncer de Mama & Rt & $\begin{array}{l}\text { Radiodermite crônica } \\
\text { com infecção } \\
\text { secundária }\end{array}$ & $\begin{array}{l}21 \text { dias de } \\
\text { uso }\end{array}$ \\
\hline 28 & E.JARD & $\mathrm{F}$ & 34 & II & Câncer de Mama & $\mathrm{Qt}+\mathrm{Rt}+\mathrm{Ht}$ & Infecção em mama & PS \\
\hline 29 & L.AGUI & $\mathrm{F}$ & 68 & III & Câncer de Mama & Rt & Xerose & $\begin{array}{l}21 \text { dias de } \\
\text { uso }\end{array}$ \\
\hline 30 & D.RIBE & $\mathrm{F}$ & 41 & II & Câncer de Mama & $Q t+R t$ & $\begin{array}{c}\text { Melasma em face; } \\
\text { hipercromia residual }\end{array}$ & $\begin{array}{l}21 \text { dias de } \\
\text { uso }\end{array}$ \\
\hline 31 & A.SANT & $\mathrm{F}$ & 36 & I & Osteossarcoma & $\mathrm{Qt}+\mathrm{Ht}$ & $\begin{array}{c}\text { Onicomicose; eflúvio } \\
\text { telógeno }\end{array}$ & $\begin{array}{l}35 \text { dias de } \\
\text { uso }\end{array}$ \\
\hline 32 & E.VIEI & $M$ & 65 & III & Câncer de Cólon & $\mathrm{Qt}+\mathrm{Rt}+$ Cirurgia & $\begin{array}{c}\text { Descamação em couro } \\
\text { cabeludo; } \\
\text { hiperqueratose plantar; } \\
\text { psoríase }\end{array}$ & $\begin{array}{l}21 \text { dias de } \\
\text { uso }\end{array}$ \\
\hline
\end{tabular}




\begin{tabular}{|c|c|c|c|c|c|c|c|c|}
\hline Prontuário & INICIAIS & SEXO & IDADE & FOTOTIPO & NEOPLASIA & $\begin{array}{c}\text { TRATAMENTO } \\
\text { ANTI-NEOPLASICO }\end{array}$ & AFECÇÕES CUTÂNEAS & $\begin{array}{l}\text { PERCURSO } \\
\text { DO } \\
\text { ESTUDO }\end{array}$ \\
\hline 33 & A.SILV & $\mathrm{F}$ & 51 & II & Câncer de Mama & Qt & $\begin{array}{c}\text { Purido; pápulas } \\
\text { eritematosas; placas } \\
\text { deprimidas atróficas } \\
\text { em couro cabeludo; } \\
\text { exulceração em região } \\
\text { de mama }\end{array}$ & $\begin{array}{l}35 \text { dias de } \\
\text { uso }\end{array}$ \\
\hline 34 & E.SILV & $\mathrm{F}$ & 74 & IV & Câncer de Cólon & $\mathrm{Qt}+\mathrm{Ht}$ & $\begin{array}{l}\text { Hiperqueratose em } \\
\text { calcâneo; xerose; } \\
\text { fotossensibilidade; } \\
\text { eczema nasal; eflúvio } \\
\text { anágeno; erupção } \\
\text { acneiforme; eritema }\end{array}$ & $\begin{array}{l}21 \text { dias de } \\
\text { uso }\end{array}$ \\
\hline 35 & J.TAVA & $\mathrm{F}$ & 62 & III & Câncer de Útero & $Q t+R t$ & $\begin{array}{l}\text { Escabiose; xerose e } \\
\text { prurido; pápulas; } \\
\text { onicomicose }\end{array}$ & PS \\
\hline 36 & J.NUS & M & 73 & I & $\begin{array}{l}\text { Câncer Espino- } \\
\text { Celular de Pele }\end{array}$ & Rt & $\begin{array}{l}\text { Radiodermite grau III } \\
\text { em região cervical } \\
\text { esquerda; eritema }\end{array}$ & $\begin{array}{l}35 \text { dias de } \\
\text { uso }\end{array}$ \\
\hline 37 & R.TEIX & $\mathrm{F}$ & 51 & II & Câncer de Mama & Qt & $\begin{array}{c}\text { Alteração de } \\
\text { pigmentação das unhas }\end{array}$ & $\begin{array}{l}35 \text { dias de } \\
\text { uso }\end{array}$ \\
\hline 38 & V.LUCI & $\mathrm{F}$ & 66 & II & Melanoma & Qt & $\begin{array}{l}\text { Cisto folicular; cisto } \\
\text { sebáceo; eczema; } \\
\text { oninfecção }\end{array}$ & $\begin{array}{l}35 \text { dias de } \\
\text { uso }\end{array}$ \\
\hline 39 & J.XAVI & $\mathrm{F}$ & 71 & II & Câncer de Reto & Qt & $\begin{array}{l}\text { Placa eritematosa } \\
\text { papulosa em face; } \\
\text { fotossensibilidade; } \\
\text { escabiose }\end{array}$ & PS \\
\hline 40 & J.PERE & M & 65 & IV & $\begin{array}{l}\text { Leiomiossarcoma de } \\
\text { coxa direita }\end{array}$ & Rt + Cirurgia & $\begin{array}{l}\text { Xerose cutânea; } \\
\text { rachadura nos pés; } \\
\text { prurido na lesão } \\
\text { cirúrgica }\end{array}$ & $\begin{array}{l}35 \text { dias de } \\
\text { uso }\end{array}$ \\
\hline
\end{tabular}

EX - Exclusão; PS - Perda de Seguimento; Qt - Quimioterapia; Rt - Radioterapia; Ht - Hormonioterapia 